\title{
Mortalidade Materna sob a ótica racial: uma revisão narrativa
}

\author{
Maternal Mortality from a racial perspective: a narrative review \\ Mortalidad Materna desde una perspectiva racial: una revisión narrativa
}

Recebido: 17/12/2021 | Revisado: 26/12/2021 | Aceito: 29/12/2021 | Publicado: 30/12/2021

\author{
Andreza Ramos Seixas dos Santos \\ ORCID: https://orcid.org/0000-0003-2450-3641 \\ Universidade Veiga de Almeida, Brasil \\ E-mail: andreza.raseixas@gmail.com \\ Abilene do Nascimento Gouvêa \\ ORCID: https://orcid.org/0000-0002-3033-5069 \\ Universidade Veiga de Almeida, Brasil \\ E-mail: abilenegouvea@gmail.com \\ Patrícia da Costa Teixeira \\ ORCID: https://orcid.org/0000-0002-1338-6091 \\ Universidade Veiga de Almeida, Brasil \\ E-mail: patrícia.teixeira@uva.br
}

\begin{abstract}
Resumo
O racismo e a discriminação racial são comuns no cotidiano brasileiro e, na área da saúde, pode levar a negligências e comprometimentos no bem-estar físico e psíquico do indivíduo e em determinadas situações pode causar o óbito. A saúde da mulher negra, e em específico da gestante, não está distante disso quando comparado a assistência que as mulheres brancas recebem. Objetivos: Identificar diferentes perfis de mortalidade materna nas literaturas e como objetivos específicos analisar literaturas com o perfil sociodemográfico de mortalidade materna, região e as principais causas de óbito. Método: Pesquisa de revisão narrativa, de análise qualitativa. Foi realizada na base de dados Biblioteca virtual em saúde (BVS), PubMed e Scientific Electronic Library Online (Scielo), por meio dos Descritores em Ciências da Saúde (DeCS): "Mortalidade materna", "Enfermagem obstétrica", "Serviços de Saúde MaternoInfantil", "Racismo", "Assistência Integral à Saúde". Resultado: Dos 24 selecionados, apenas 7 bibliografias continham as variáveis para responder o objetivo deste estudo, com 5 sendo oriundas do nordeste. Foi identificado taxa de óbitos frequentes em mulheres da cor parda, escolaridade com fundamental completo, faixa etária dos 20 aos 39 e causa obstétrica direta síndromes hipertensivas, enquanto as indiretas não foram especificadas. Conclusão: É necessário que haja um maior investimento em pesquisas dessa temática devido a gravidade da situação e a quantidade baixa de literaturas. É possível que esses dados na raça parda sejam bem mais elevados do que na raça preta devido a autodeclaração, infelizmente ainda há uma certa resistência em se declarar preto e isso reflete nos dados avaliados.
\end{abstract}

Palavras-chave: Assistência integral à saúde; Enfermagem obstétrica; Mortalidade materna; Racismo.

\begin{abstract}
Racism and racial discrimination are common in Brazilian daily life and, in the area of health, it can lead to negligence and compromise in the individual's physical and mental well-being and, in certain situations, it can cause death. The health of black women, and specifically of pregnant women, is not far from this when compared to the assistance that white women receive. Objectives: To identify literature with the sociodemographic profile of maternal mortality, region and main causes of death. Method: Narrative review research, qualitative analysis. It was performed in the Virtual Health Library (BVS), PubMed and Scientific Electronic Library Online (Scielo) database, through the Health Sciences Descriptors (DeCS): "Maternal mortality", "Obstetrical Nursing", "Health Services Maternal and Child", "Racism", "Comprehensive Health Care". Result: Of the 24 selected, only 7 bibliographies contained the variables to answer the objective of this study, with 5 coming from the northeast. A frequent death rate was identified in brown women, schooling with complete elementary school, age group from 20 to 39 and direct obstetric cause of hypertensive syndromes, while indirect causes were not specified. Conclusion: There needs to be greater investment in research on this topic due to the seriousness of the situation and the low amount of literature. It is possible that these data in the brown race are much higher than in the black race due to self-declaration, unfortunately there is still some resistance in declaring oneself black and this reflects in the data evaluated.
\end{abstract}

Keywords: Comprehensive health care; Obstetric nursing; Maternal mortality; Racism. 


\begin{abstract}
Resumen
El racismo y la discriminación racial son habituales en la vida cotidiana brasileña y, en el ámbito de la salud, pueden provocar negligencia y comprometer el bienestar físico y mental del individuo y, en determinadas situaciones, provocar la muerte. La salud de las mujeres negras, y en particular de las embarazadas, no está lejos de esto si se compara con la asistencia que reciben las mujeres blancas. Objetivos: Identificar diferentes perfiles de mortalidad materna en la literatura y como objetivos específicos analizar la literatura con el perfil sociodemográfico de la mortalidad materna, región y principales causas de muerte. Método: Investigación de revisión narrativa, análisis cualitativo. Se realizó en la base de datos de la Biblioteca Virtual en Salud (BVS), PubMed y Biblioteca Electrónica Científica en Línea (Scielo), a través de los Descriptores de Ciencias de la Salud (DeCS): "Mortalidad materna", "Enfermería obstétrica", "Servicios de salud maternoinfantil", "Racismo", "Atención Integral a la Salud". Resultado: De las 24 seleccionadas, solo 7 bibliografías contenían las variables para responder al objetivo de este estudio, de las cuales 5 procedían del noreste. Se identificó una tasa de mortalidad frecuente en mujeres morenas, escolaridad con primaria completa, grupo de edad de 20 a 39 años y causa obstétrica directa de síndromes hipertensivos, mientras que no se especificaron las causas indirectas. Conclusión: Se necesita una mayor inversión en la investigación sobre este tema debido a la gravedad de la situación y la escasa bibliografía. Es posible que estos datos en la raza morena sean mucho más altos que en la raza negra debido a la autodeclaración, lamentablemente aún existe cierta resistencia en declararse negro y esto se refleja en los datos evaluados.
\end{abstract}

Palabras clave: Atención integral a la salud; Enfermería obstétrica; Mortalidad maternal; Racismo.

\title{
1. Introdução
}

De acordo com Viana, Novaes e Calderon (2011), a Organização Mundial de Saúde (OMS) definiu a mortalidade materna (MM) como a que acontece durante a gestação ou no puerpério, ou seja, período de 42 dias. Ainda de acordo com a OMS, essa classificação não se enquadra para óbitos de causas acidentais ou incidentais, apenas para causas relacionadas ou agravadas pela gravidez.

Os comitês de MM do Ministério da Saúde (MS) definem em causas diretas e indiretas, tendo a direta como complicações obstétricas, durante gravidez, parto ou puerpério, relacionadas a intervenções, omissões, tratamento incorreto ou a uma cadeia de eventos, resultantes de qualquer uma dessas causas. (Viana et al., 2011) e causa indireta as doenças preexistentes ou que foram desenvolvidas nesse período gravídico-puerperal.

Desde 1990, vários países subsaarianos têm reduzido pela metade a mortalidade materna. Em outras regiões, como a Ásia e o Norte da África, os progressos têm sido ainda maiores. Entre 1990 e 2015, a taxa de mortalidade materna global diminuiu apenas 2,3\% ao ano. (Organização Pan-Americana da Saúde [OPAS], 2020). No Brasil, com a criação de políticas e ações públicas para a saúde das mulheres, a mortalidade também entrou em evidência e a partir do Sistema de Informação sobre Mortalidade, do ministério da saúde (SIM/MS), começou-se a ter um certo controle desses óbitos. De acordo com Laurenti, Helena \& Léa, 2004, pode-se dizer que a cobertura do SIM/MS seja por volta de $85 \%$.

Com o passar dos anos e o avanço da tecnologia é notável que tenha ocorrido um declínio na taxa de mortalidade materna, quando comparado a questão raça/cor. De acordo com o Manual de Gestão para Implementação da Política Nacional de Saúde Integral da População negra [PNSIPN] (MS, 2018) em 2000, 16,6\% tiveram sua cor/ raça ignorada, diferente de cinco anos depois, onde a porcentagem foi para 8,3\% e em 2012 se atingiu 4,5\%. Ou seja, em doze anos essa taxa diminuiu pelo triplo do apresentado em 2000.

Ainda assim, ainda de acordo com o Manual de Gestão para Implementação da Política Nacional de Saúde Integral da População negra (MS, 2018) em 2012 num total de mil e quinhentas e oitenta e três mortes, 60\% eram de mulheres negras. Mesmo com avanços, a porcentagem ainda é maior nas mulheres negras e isso pode se dar por 
diversos fatores sejam eles doenças preexistentes, falta de recursos para uma boa assistência de saúde ou até mesmo negligência médica.

Sabendo que são direitos básicos, garantidos em Lei, uma boa assistência e qualidade de saúde a todos os cidadãos, independentemente de sua cor/raça e condição social, mas diante de dados de óbitos relativamente maiores em mulheres negras, surgiu a questão norteadora: $\mathrm{O}$ que as literaturas dizem sobre o perfil racial de mortalidade materna e quais suas principais causas?

Ciente deste cenário e objetivando contribuir para pesquisas relacionadas a presente discussão, surgiu o interesse no tema, a fim de identificar literaturas que dizem sobre o perfil de mortalidade materna. Sua relevância se dá no âmbito da saúde, social e para a academia, onde foram analisados estudos sobre o perfil de óbito materno relacionado a sua questão sociodemográfica.

Diante do exposto, a pesquisa visa como objetivo geral identificar diferentes perfis de mortalidade materna nas literaturas e como objetivos específicos analisar literaturas com o perfil sociodemográfico de mortalidade materna, região e as principais causas de óbito.

\section{Metodologia}

Trata-se de um estudo do tipo revisão narrativa de literatura (RNL). Não utiliza critérios explícitos e sistemáticos para a busca e análise crítica da literatura. A busca pelos estudos não precisa esgotar as fontes de informações. Não aplica estratégias de busca sofisticadas e exaustivas. (Universidade Estadual Paulista em Franca [UNESP], 2015).

Para dar início a resposta da questão norteadora, foi feito o levantamento dos artigos nos meses outubro e novembro de 2021, sendo utilizado o operador booleano "AND” para busca simultânea nos seguintes descritores: "Mortalidade materna", "Enfermagem and obstétrica and mortalidade", "Serviços de Saúde Materno-Infantil", "Racismo", “Assistência Integral à Saúde”. Acessados nas bases de dados BVS, PubMed e Scielo.

A seleção foi feita partindo dos critérios de inclusão de obras do período de 2016 a 2021, no idioma português, título e conteúdo com maior relevância sobre a temática. Já os de exclusão foram os repetidos nas bases de dados, fora do período de inclusão e os estudos ao qual não respondiam os objetivos da questão norteadora.

A busca foi realizada utilizando os filtros Base de dados Enfermagem (BDENF) e Literatura LatinoAmericana e do Caribe em Ciências da Saúde (LILACS), texto completo e período de 2016 a 2021. Foram encontrados ao todo 697 artigos através dos descritores selecionados nas três bases de dados. Seguindo os critérios de inclusão e exclusão, 52 foram separados de acordo com o título, porém 10 foram excluídos por repetição e 18 foram desconsiderados por não atender as propostas da pesquisa. Sendo assim, foram selecionadas 24 publicações de acordo com título, resumo e objetivos para compor este estudo.

Sucessivamente, foi feito uma tabela com os seguintes aspectos pertinentes: título, autor, ano, tipo de estudo, coleta de dados e região do estudo.

A apresentação dos resultados e a discussão dos dados analisados foram feitas de forma descritiva e narrativa, de forma a contribuir com a comunidade científica. 


\section{Resultados e Discussão}

Foram analisadas 24 literaturas, sendo descartadas 6 com fuga parcial do tema, 2 não traçando o perfil racial, 3 fora da língua de inclusão, 3 repetidos e 2 fora do período proposto. Sobrando então 7 literaturas para compor esse estudo, sendo nenhuma revisão bibliográfica.

As pesquisas que compõem o tema em questão foram abordadas no início do século, sendo o dado mais recente do ano de 2017, porém somente neste ano que começou a ser coletado nos dados dos serviços públicos a questão raça/cor, de acordo com a autodeclaração do cliente. Dessa forma, é possível que muitos dados estejam subnotificados devido à falta de dados registrados e a mudança na autodeclaração. "De acordo com o Censo 2010 do Instituto Brasileiro de Geografia e Estatística (IBGE), negras e negros constituem mais da metade da população brasileira (50,7\%). Dados mais recentes apontam que, em 2015, 53,9\% das pessoas se declararam de cor ou raça preta ou parda." (Carvalho, Meirinho, 2020). Outro fato pertinente nesta pesquisa é a região frequente das literaturas, a qual cinco são do nordeste, uma do sudeste e uma do centro-oeste, e por mais que as literaturas sejam de diferentes estados e regiões, com estruturas, miscigenação e desenvolvimento distintos, a taxa de óbito da raça/cor preta e parda é prevalente nos estudos.

A forma que os dados foram coletados foi em $80 \%$ pelo SIM e $20 \%$ nas Fichas de Investigação de Óbito Materno. Porém, os autores de 'Distribuição da mortalidade materna no estado da Paraíba no período de 2007 a 2016', 'Perfil sociodemográfico e clínico de mortalidade materna' e 'Diagnóstico da situação de morte materna' relatam uma possível subnotificação desses dados por se tratar de um sistema secundário e pela omissão de informações na declaração de óbito. Ao referir o tipo de estudo se tem uma predominância do tipo descritivo, onde segundo Costa, Barreto (2003) os estudos descritivos determinam a distribuição das doenças ou semelhantes, de acordo com as características dos indivíduos. Apenas duas literaturas não utilizaram esse método, como pode ser observado na Tabela 1. 
Tabela 1: Caracterização Das Literaturas Quanto Ao Autor, Ano, Tipo De Estudo, Base De Dados E Região.

\begin{tabular}{|c|c|c|c|c|c|}
\hline Título & Autor & Ano & Tipo de estudo & Coleta de dados & Região do estudo \\
\hline $\begin{array}{l}\text { Mortalidade materna segundo } \\
\text { raça/cor, em Mato Grosso do Sul, } \\
\text { Brasil, de } 2010 \text { a } 2015\end{array}$ & Pícoli, Cazola, Lemos & 2017 & $\begin{array}{l}\text { Epidemiológico, descritivo } \\
\text { e retrospectivo }\end{array}$ & $\begin{array}{ll}\text { Sistemas } & \text { de } \\
\text { Informações } & \text { de } \\
\text { Mortalidade e } & \text { de } \\
\text { Nascidos Vivos. } & \end{array}$ & $\begin{array}{l}\text { Mato Grosso do } \\
\text { Sul }\end{array}$ \\
\hline $\begin{array}{l}\text { Perfil e distribuição espacial da } \\
\text { mortalidade materna }\end{array}$ & $\begin{array}{l}\text { Ruas, Quadros, Rocha, } \\
\text { Rocha, Neto, Piris, Rios, } \\
\text { Pereira, Ribeiro, Leão }\end{array}$ & 2020 & $\begin{array}{l}\text { Descritivo, transversal, } \\
\text { documental e quantitativo }\end{array}$ & $\begin{array}{l}\text { Fichas de Investigação } \\
\text { de Óbito Materno do } \\
\text { Comitê de Mortalidade } \\
\text { Materna. }\end{array}$ & Minas Gerais \\
\hline $\begin{array}{l}\text { Perfil sociodemográfico e } \\
\text { assistencial da morte materna em } \\
\text { Recife, 2006-2017: estudo } \\
\text { descritivo }\end{array}$ & $\begin{array}{l}\text { Carvalho, } \begin{array}{r}\text { Frias, } \\
\text { Frutuoso, }\end{array} \\
\text { Pereira, Barreto, }\end{array}$ & 2020 & Descritivo & $\begin{array}{l}\text { SIM, fichas } \text { de } \\
\text { investigação e fichas- } \\
\text { síntese de óbitos } \\
\text { maternos. }\end{array}$ & Pernambuco \\
\hline $\begin{array}{l}\text { Diagnóstico da situação de morte } \\
\text { materna }\end{array}$ & $\begin{array}{l}\text { Silva, Monteiro, Freitas, } \\
\text { Barros, Guimarães, Melo }\end{array}$ & 2019 & $\begin{array}{l}\text { Transversal, com } \\
\text { delineamento } \\
\text { observacional e } \\
\text { retrospectivo, de } \\
\text { abordagem quantitativa }\end{array}$ & SIM & Paraíba \\
\hline $\begin{array}{l}\text { Perfil sociodemográfico e clínico } \\
\text { de mortalidade materna }\end{array}$ & $\begin{array}{l}\text { Gomes, Vieira, Mistura, } \\
\text { Andrade, Barbosa, Lira, } \\
\text { Ferreira, Justino }\end{array}$ & 2018 & $\begin{array}{l}\text { Quantitativo, transversal e } \\
\text { descritivo }\end{array}$ & SIM & Pernambuco \\
\hline $\begin{array}{l}\text { Caracterização dos óbitos } \\
\text { maternos em três regiões de saúde } \\
\text { do centro-oeste norte baiano. }\end{array}$ & $\begin{array}{l}\text { Coelho, Andrade, Sena, } \\
\text { Costa, Bittencourt }\end{array}$ & 2016 & Descritivo, transversal & SIM & Bahia \\
\hline $\begin{array}{l}\text { Distribuição da mortalidade } \\
\text { materna no estado da Paraíba no } \\
\text { período de } 2007 \text { a } 2016\end{array}$ & Batista & 2020 & Documental e quantitativa & SIM & Paraíba \\
\hline
\end{tabular}

Fonte: Autores (2021).

Conforme o tipo de estudo apresentado, foram encontrados como delineamento para compor os estudos, variáveis para se chegar ao objetivo de situação de MM. As variáveis encontradas foram cor/raça, idade, escolaridade, causa obstétrica direta e indireta em que momento ocorreu o óbito, porém para compor esta pesquisa a variável de momento do óbito não será utilizada. Assim, através de taxas foi possível chegar a um resultado quantitativo.

A metodologia utilizada facilita a replicação de estudos sobre o tema, devido a sua fácil aplicabilidade e o sistema de dados ser de origem secundária e pública. É um fator importante pois a quantidade de literaturas recentes sobre o tema ainda é pouca para a gravidade do tema em questão.

Foi possível identificar o tipo de raça em todos os estudos apresentados, assim como a idade frequente e a causa obstétrica direta, porém se tratando da causa obstétrica indireta nem todos especificaram exatamente a causa, apenas a quantidade. A variável escolaridade não foi possível identificar em uma literatura, como apresentado na Tabela 2. 
Tabela 2: Exemplificação de quais estudos abordaram as variáveis pré-determinadas.

\begin{tabular}{|c|c|c|c|c|c|}
\hline Autor & Cor/Raça & Idade & Causa Direta & Causa Indireta & Escolaridade \\
\hline Pícoli, Cazola, Lemos & SIM & SIM & SIM & NÃO & SIM \\
\hline $\begin{array}{l}\text { Ruas, Quadros, Rocha, Rocha, } \\
\text { Neto, Piris, Rios, Pereira, } \\
\text { Ribeiro, Leão }\end{array}$ & SIM & SIM & SIM & $\mathrm{NÃO}$ & SIM \\
\hline $\begin{array}{l}\text { Carvalho, Frias, Lemos, } \\
\text { Frutuoso, Figueirôa, Pereira, } \\
\text { Barreto, Vidal }\end{array}$ & SIM & SIM & SIM & NÃO & NÃO \\
\hline $\begin{array}{l}\text { Silva, Monteiro, Freitas, } \\
\text { Barros, Guimarães, Melo }\end{array}$ & SIM & SIM & SIM & SIM & SIM \\
\hline $\begin{array}{l}\text { Gomes, Vieira, Mistura, } \\
\text { Andrade, Barbosa, Lira, } \\
\text { Ferreira, Justino }\end{array}$ & SIM & SIM & SIM & $\mathrm{NÃO}$ & SIM \\
\hline $\begin{array}{l}\text { Coelho, Andrade, Sena, Costa, } \\
\text { Bittencourt }\end{array}$ & SIM & SIM & SIM & SIM & SIM \\
\hline Batista & SIM & SIM & SIM & $\mathrm{NÃO}$ & SIM \\
\hline Pícoli, Cazola, Lemos & SIM & SIM & SIM & NÃO & SIM \\
\hline
\end{tabular}

Fonte: Autores (2021).

Com relação a variável raça/cor buscava-se encontrar as seguintes: Amarela, Branca, Indígena, Parda, Preta e Não identificado. Com isso, obteve-se o resultado de duas literaturas considerando a raça amarela e uma unificando a raça amarela com a branca e a parda com a preta, com relação a indígena foram três achados num total das sete literaturas. As raças branca e preta estiveram presentes em todos os estudos e a parda em cinco. Apenas na primeira literatura não apresentou nenhum dado referente a não informado. Amarela $n=3$; Branca $n=7$; Indígena n=3; Parda n=5; Preta n=7; Não informado n=6.

Diante disso, foi constatado em cinco estudos a prevalência de óbitos na raça/cor parda e duas na preta, sendo as duas pretas em uma bibliografia que só considerou brancas e pretas e outra que uniu a preta com a parda.

Para considerar a taxa de óbitos, Pícoli, Cazola, Lemos e Ruas, Quadros, Rocha, Rocha, Neto, Piris, Rios, Pereira, Ribeiro, Leão utilizaram a razão de mortalidade materna (RMM) com a de nascidos vivos para aquela época, já os demais tiraram apenas a porcentagem de total investigados. Considerando que os estudos foram de diferentes regiões e de diferentes formas de cálculo, dificultou a estimação da porcentagem total de óbitos de pardas, mas é possível observar separadamente a porcentagem dos estudos de acordo com a Tabela 3. 
Tabela 3: Caracterização do quantitativo de óbitos relacionados com a raça prevalente na obra e a sua porcentagem.

\begin{tabular}{|l|l|l|l|}
\hline $\begin{array}{l}\text { Mortalidade materna segundo raça/cor, em Mato Grosso do Sul, } \\
\text { Brasil, de } 2010 \text { a } 2015\end{array}$ & Parda & 166 Óbitos & $92-55,4 \%$ \\
\hline Perfil e distribuição espacial da mortalidade materna & Parda & 31 Óbitos & $22-70,9 \%$ \\
\hline $\begin{array}{l}\text { Perfil sociodemográfico e assistencial da morte materna em Recife, } \\
\text { 2006-2017: estudo descritivo* }\end{array}$ & Preta $^{(*)}$ & 171 Óbitos & $117-68,4 \%$ \\
\hline Diagnóstico da situação de morte materna & Preta/Parda $^{(*)}$ & 259 Óbitos & $201-59,11 \%$ \\
\hline Perfil sociodemográfico e clínico de mortalidade materna & Parda & 1907 Óbitos & $1130-59,25 \%$ \\
\hline $\begin{array}{l}\text { Caracterização dos óbitos maternos em três regiões de saúde do } \\
\text { centro-norte baiano }\end{array}$ & Preta ${ }^{(*)}$ & 113 Óbitos & $85-75,2 \%$ \\
\hline $\begin{array}{l}\text { Distribuição da mortalidade materna no estado da Paraíba no } \\
\text { período de } 2007 \text { a } 2016\end{array}$ & Parda & 324 Óbitos & $241-74,4 \%$ \\
\hline
\end{tabular}

${ }^{(*)}$ Foram consideradas apenas pretas e brancas. ${ }^{(* *)}$ Foi considerado a raça preta e parda com o mesmo quantitativo. Fonte: Autores (2021).

Nas causas, a obstétrica direta a prevalência é das síndromes hipertensivas sem e com proteinúria, sendo encontrado em seis estudos, e uma literatura abordando a complicação do trabalho de parto e parto como a principal causa de óbito. Nas indiretas os estudos que apresentaram, a frequência foi a de doenças do aparelho circulatório e outras doenças da mãe.

Se tratando das variáveis da idade e escolaridade, foi observado uma prevalência de óbitos na faixa etária dos 20 a 39 anos e escolaridade de fundamental completo, como pode ser observado na Tabela 4.

Tabela 4: Relação do autor e as variáveis identificadas com prevalência na obra.

\begin{tabular}{|c|c|c|c|c|c|}
\hline Autor & Raça/Cor & Idade & Escolaridade & Causas Diretas & Causas Indiretas \\
\hline Pícoli, Cazola, Lemos & Parda & $40-49$ & Sem Escolaridade & $\begin{array}{l}\text { Complicações Do Trabalho } \\
\text { De Parto E Parto }\end{array}$ & Não Especificado \\
\hline $\begin{array}{l}\text { Ruas, Quadros, Rocha, Rocha, } \\
\text { Neto, Piris, Rios, Pereira, } \\
\text { Ribeiro, Leão }\end{array}$ & Parda & $20-34$ & Fundamental Incompleto & $\begin{array}{l}\text { Síndrome Hipertensiva Da } \\
\text { Gestação E Neoplasias }\end{array}$ & Não Especificado \\
\hline $\begin{array}{lll}\text { Carvalho, Frias, } & \text { Lemos, } \\
\text { Frutuoso, Figueirôa, } & \text { Pereira, } \\
\text { Barreto, Vidal } & \end{array}$ & Preta $^{(*)}$ & $20-39$ & Fundamental Ou Médio & Hipertensão Arterial & Aparelho Circulatório \\
\hline $\begin{array}{l}\text { Silva, Monteiro, Freitas, } \\
\text { Barros, Guimarães, Melo }\end{array}$ & Preta/Parda ${ }^{(* *)}$ & $20-39$ & - & Hipertensão Arterial & Não Especificado \\
\hline $\begin{array}{l}\text { Gomes, } \quad \text { Vieira, } \\
\text { Andrade, } \quad \text { Barbosa, } \\
\text { Ferreira, Justino }\end{array}$ & Parda & $20-29$ & 8-11 Anos & Eclâmpsia & $\begin{array}{lll}\text { Outras } & \text { Doenças } & \text { Das } \\
\text { Mãe } & & \end{array}$ \\
\hline $\begin{array}{l}\text { Coelho, Andrade, Sena, Costa, } \\
\text { Bittencourt }\end{array}$ & $\operatorname{Preta}^{(*)}$ & $20-29$ & 4-7 Anos & $\begin{array}{l}\text { Pré-Eclâmpsia } \\
\text { Eclâmpsia }\end{array}$ & Não Especificado \\
\hline Batista & Parda & $30-39$ & Ignorado & Síndromes Hipertensivas & Não Especificado \\
\hline
\end{tabular}

${ }^{(*)}$ Foram consideradas apenas pretas e brancas. ${ }^{(* *)}$ Foi considerado a raça preta e parda com o mesmo quantitativo. Fonte: Autores (2021). 
Apesar das diferentes localidades e tipo de estudo, mesmo sem a possibilidade de generalizar as taxas, todos ressaltaram a discrepância nos dados quando comparado o óbito com mulheres brancas e não brancas. É possível correlacionar esse achado com a baixa escolaridade, onde o acesso a informações sobre saúde é mais ineficaz fazendo com que aumente as causas diretas, ao qual era possível evitar. O recorte étnico-racial expõe as desigualdades da sociedade, a condição socioeconômica e a iniquidade no acesso aos serviços de saúde dirigidos a essa população. (Carvalho, Frias, Lemos, Frutuoso, Figueirôa, Pereira, Barreto, Vidal, 2020).

É importante ressaltar que ainda há um número relativamente alto de pessoas que se autodeclaram parda, dessa forma é possível identificar uma maior quantidade de óbitos dessa cor.

Reconhecemos que este fator traz importantes limitações nas interpretações dos resultados da análise e pode estar diretamente relacionado ao declínio das taxas de mortalidade de mulheres negras e, consequentemente, de mulheres que se autodeclararam brancas no preenchimento da Declaração de Nascido Vivo, logo após o parto pelo profissional de saúde que prestou assistência ao parto hospitalar ou domiciliar. (Carvalho \& Meirinho, 2020)

\section{Conclusão}

De modo geral, é possível observar que há uma deficiência de estudos sobre a temática, principalmente com dados recentes, onde nota-se que para a construção da introdução deste estudo, as referências utilizadas foram abaixo do período estabelecido para compor os resultados. Além de ter uma menor quantidade de literaturas traçando o perfil sociodemográfico no eixo sul-sudeste e norte do país. Essa avaliação territorial é importante devido ao Brasil ser um país miscigenado e com grandes disparidades regionais, para que assim seja possível intervir e chegar ao objetivo do desenvolvimento sustentável, onde tem como meta da mortalidade materna 30 a cada 100 mil nascidos vivos até 2030, além de realizar comparações quanto a queda, aumento e/ou oscilações e onde é necessária uma atenção maior para que essas taxas caiam.

Enfatiza-se, portanto, a necessidade de mais pesquisas sobre o assunto seja de forma exploratória ou de revisão, para que assim haja mais contribuições para a sociedade e a academia científica entenderem a gravidade e intervir nessa problemática.

É possível afirmar que os objetivos propostos foram atendidos, assim como a questão norteadora, onde foi possível identificar a frequência de óbitos na questão raça, a escolaridade, a idade e as causas sendo elas obstétricas diretas ou não.

\section{Referências}

Alves De Souza, C., Monteiro Ramos, M. A., \& Almeida Vasconcelos, M. D. C. (2020). O quesito raça/cor e a política nacional de saúde integral da população negra. In XIV colóquio internacional "educação e contemporaneidade". Grupo de Estudos e Pesquisas "Educação e Contemporaneidade".

Batista, H. M. T. (2019). Distribuição da mortalidade materna no estado da paraíba no período de 2007 a 2016. Revista de Epidemiologia e Controle de Infecção, 9(4).

Botelho, N. M., Silva, I. F. M. M., Tavares, J. R., \& Lima, L. O. (2014). Causas de morte materna no estado do pará, brasil. Revista Brasileira de Ginecologia e Obstetrícia, 36(7), 290-295.

Brasil (2018). Manual de Gestão Para Implementação da Política Nacional de Saúde Integral da População Negra. Ministério da Saúde, 1, 20. https://bvsms.saude.gov.br/bvs/publicacoes/manual_gestao_implementacao_politica_nacional.pdf

Brasil (2017), Política Nacional de Saúde Integral da População Negra: Uma política do SUS. Ministério da Saúde, 3. https://bvsms.saude.gov.br/bvs/publicacoes/politica_nacional_saude_populacao_negra_3d.pdf 
Camargo, L. M. A., Silva, R. P. M., \& Meneguetti, D. U. D. O. (2019). Research methodology topics: Cohort studies or prospective and retrospective cohort studies. Journal of Human Growth and Development, 29(3), 433-436.

Campos Mendes Silva, S., Araújo Monteiro, E., de Mendonça Faustino e Freitas, W., Gonçalves de Barros, A., Maria Cardoso Guimarães, C., \& de Almeida Melo, S. (2019). Diagnóstico da situação de morte materna. Revista Brasileira em Promoção da Saúde, 32, 1-11.

Carvalho, D., \& Meirinho, D. (2020). O quesito cor/raça: Desafios dos indicadores raciais de mortalidade materna como subsídio ao planejamento de políticas públicas em saúde. Revista Eletrônica de Comunicação, Informação e Inovação em Saúde, 14(3).

Carvalho, P. I. d., Frias, P. G. d., Lemos, M. L. C., Frutuoso, L. A. L. d. M., Figueirôa, B. d. Q., Pereira, C. C. d. B., Barreto, I. d. C., \& Vidal, S. A. (2020). Perfil sociodemográfico e assistencial da morte materna em Recife, 2006-2017: Estudo descritivo*. Epidemiologia e Serviços de Saúde, 29(1).

Coelho, V. C., Andrade, M. S., De Sena, C. D., Costa, L. E. L., \& Bittencourt, I. S. (2016). Caracterização dos óbitos maternos em três regiões de saúde do centro-norte baiano. Cogitare Enfermagem, 21(1).

Dias Armada e Silva, H. C., Bernardo da Silva, M. R., De Sousa Barros, F. R., Ribeiro Soares de Souza, D., Da Silva de Medeiros, C., \& Bernardo da Silva, R. (2020). Análise da mortalidade materna como indicador de gestão do enfermeiro na atenção primária à saúde. Saúde Coletiva (Barueri), (53), $2460-2469$.

Laurenti, R., Jorge, M. H. P. d. M., \& Gotlieb, S. L. D. (2004). A mortalidade materna nas capitais brasileiras: Algumas características e estimativa de um fator de ajuste. Revista Brasileira de Epidemiologia, 7(4), 449-460.

Lima-Costa, M. F., \& Barreto, S. M. (2003). Tipos de estudos epidemiológicos: Conceitos básicos e aplicações na área do envelhecimento. Epidemiologia e Serviços de Saúde, 12(4).

M, N.-P., \& J, R. F. (2021). Mortalidade materna no Brasil: Uma tragédia evitável. Anais Da Academia Nacional De Medicina, 192(Especial), 95-101.

Martins. (2018). Estudos de revisão de literatura. Trabalho apresentado no Curso de Acesso à Informação Científica e Tecnológica em Saúde. Modalidade: Qualificação. Fundação Oswaldo Cruz.

Pícoli, R. P., Cazola, L. H. d. O., \& Lemos, E. F. (2017). Maternal mortality according to race/skin color in Mato Grosso do Sul, Brazil, from 2010 to 2015. Revista Brasileira de Saúde Materno Infantil, 17(4), 729-737.

Rodrigues, D. Lima, C. Marcela, A. Rezende, T. Ventura, K. (2016). Análise dos fatores intervenientes da mortalidade materna. Revista Enfermagem obstétrica.

Ruas, C. A. M., Quadros, J. F. C., Rocha, J. F. D., Rocha, F. C., Andrade Neto, G. R. d., Piris, Á. P., Rios, B. R. M., Pereira, S. G. S., Ribeiro, C. D. A. L., \& Leão, G. M. M. S. (2020). Profile and spatial distribution on maternal mortality. Revista Brasileira de Saúde Materno Infantil, $20(2), 385-396$.

Saúde materna - OPAS/OMS | Organização Pan-Americana da Saúde. (n.d.). PAHO/WHO | Pan American Health Organization. https://www.paho.org/pt/node/63100

Silva, B. G. C. d., Lima, N. P., Silva, S. G. d., Antúnez, S. F., Seerig, L. M., Restrepo-Méndez, M. C., \& Wehrmeister, F. C. (2016). Mortalidade materna no Brasil no período de 2001 a 2012: Tendência temporal e diferenças regionais. Revista Brasileira de Epidemiologia, 19(3), 484-493.

Teodoro, M. S., Santos, P. H. E. d., Souza, M. C. d., Riskalla, D. B., Barbosa, J. V. B., Guimarães, B. D. G., \& Passos, M. Z. (2021). Condicionantes e características da mortalidade materna no Brasil. Revista Eletrônica Acervo Saúde, 13(4), Artigo e7050.

Universidade Estadual Paulista em Franca (2015). Tipos de Revisão de Literatura. Trabalho apresentado como normas para trabalhos acadêmicos. Faculdade de Ciências Agronômicas - Câmpus de Botucatu.

Viana, R. Novaes, M. Calderon, I. (2011). Mortalidade Materna - uma abordagem atualizada. Revista comunicação em Ciências da Saúde.

Vieira, M. C. A., Gomes, J. O., Mistura, C., Andrade, G. G. d., Araújo Vieira, K. M., Carvalho e Lira, M. O. d. S., Ferreira, M. A., \& Justino, T. M. V. (2018). Perfil sociodemográfico e clínico de mortalidade materna. Revista De Enfermagem UFPE on Line, 12(12), 3165. 